\title{
White-light spectral interferometric technique to measure a nonlinear phase function of a thin-film structure
}

\author{
P. Hlubina* , J. Luňáček, D. Ciprian \\ Department of Physics, Technical University Ostrava, 17. listopadu 15, \\ 70833 Ostrava-Poruba, Czech Republic
}

\begin{abstract}
We present a new two-step white-light spectral interferometric technique to measure a nonlinear phase function of a thin-film structure. The technique is based on recording of channeled spectra at the output of a Michelson interferometer and their processing by using a windowed Fourier transform to retrieve the phase functions. First, the phase function including the effect of a thin-film structure is retrieved. Second, the structure is replaced by a reference sample of known phase change on reflection and the corresponding phase function is retrieved. From the two functions, the nonlinear phase function of the thin-film structure is obtained. The feasibility of this simple method is confirmed in processing the experimental data for a $\mathrm{SiO}_{2}$ thin film on a Si wafer of known optical constants. Four samples of the thin film are used and their thicknesses are determined. The thicknesses obtained are compared with those resulting from reflectometric measurements, and good agreement is confirmed.
\end{abstract}

Key words: spectral interferometry, Michelson interferometer, thin film, thickness, phase change on reflection, nonlinear phase function

PACS: 07.60.Ly, 42.30.Rx, 68.55.Jk

\section{Introduction}

Optical measurement methods, based on ellipsometry [1,2], reflectometry $[3,4]$ or interferometry $[5,6]$, belong to the most important ones in research of thinfilm structures. Ellipsometric angles, reflectances and interferometric phases

* Tel.: +420-597-323-134; fax: +420-597-323-139.
Email address: petr.hlubina@vsb.cz (P. Hlubina). 
are of interest in determining the parameters and characteristics of the structures. Ellipsometric measurements performed at a single wavelength and a fixed angle of incidence provide the film thickness and optical constants [1]. Measurements by spectroscopic ellipsometry provide the results over a wide wavelength range with greater precision and accuracy [2]. Normal incidence spectroscopic reflectometry [3] or oblique incidence reflectance measurement [4] applied over a wide wavelength range is also a useful tool for the characterization of thin films. The optical method most commonly employed for micrometer-scale thickness measurements is Fourier transform white-light interferometry [5]. The technique is based on retrieving the relative phase change on reflection from a thin-film structure. The use of white-light interferometry was extended into the spectral domain [6-9] where the phase of the reflected wave, which changes as a function of wavelength [10] and layer thickness, is inscribed in the recorded spectral interferogram (channeled spectrum). Some of the techniques $[7,8]$ that are based on Fourier transforming of the channeled spectra enable a complete 3D tomographical reconstruction of a thinfilm layer. This reconstruction is possible using the retrieved time delays [7] or the spectrally resolved phases and reflectances [8].

Recently, we have used dispersive white-light spectral interferometry for measuring the thickness of a $\mathrm{SiO}_{2}$ thin film on a $\mathrm{Si}$ wafer $[11,12]$. The technique utilizes a slightly dispersive Michelson interferometer with a beam splitter cube and one of the mirrors replaced by a thin-film structure of known optical constants. The thickness of the thin film is determined from the fit of the absolute optical path difference (OPD), which was retrieved from the channeled spectrum $[11,12]$, to the theoretical one. We have also retrieved the oscillatory part of the phase change on reflection, which is referred to as the nonlinear phase [11,12], and used it for determining the thin-film thickness. However, the results suffered from the systematic phase errors due to the optical components present in the interferometer. To minimize them, a procedure with the reference measurement needs to be applied [8]. We applied a three-step procedure [13] that enabled us to determine both the effective thickness of a beam splitter cube and the phase contribution of the interferometer optical elements.

We present in this paper a new two-step white-light spectral interferometric technique to retrieve a nonlinear phase function of a thin-film structure without the need of any information about the effective thickness of a beam splitter cube and the phase contribution of the interferometer optical elements. The technique is based on recording of the channeled spectra at the output of a Michelson interferometer and their processing by using a windowed Fourier transform (WFT) to retrieve the phase functions. First, the phase function including the effect of a thin-film structure is retrieved. Second, the structure is replaced by a reference sample of known phase change on reflection and the corresponding phase function is retrieved. From the two functions, the non- 
linear phase function of the thin-film structure is obtained. The feasibility of the simple method was confirmed in processing the experimental data for a $\mathrm{SiO}_{2}$ thin film on a $\mathrm{Si}$ wafer of known optical constants. Four samples of the thin film were used and their thicknesses were determined. The thicknesses obtained were compared with those resulting from reflectometric measurements performed in the same setup [13], and good agreement was confirmed.

\section{Measurement method}

Consider the mutual interference of two beams from a broadband (white-light) source at the output of a Michelson interferometer (see Fig. 1) with a cube beam splitter of the effective thickness $t_{\text {eff }}$ [11]. We assume that the geometrical path lengths of the light rays in dispersive glass of the beam splitter are not the same for both interferometer arms and that the beam splitter can be represented by an ideal beam splitter and a plate (see a dashed element in Fig. 1) of the same dispersion and of the thickness $t_{\text {eff }}$. The interferometer comprises a mirror and a thin-film structure (a thin film on a substrate) that are characterized by the complex reflection coefficients

$$
r_{M}(\lambda)=\sqrt{R_{M}(\lambda)} \exp \left[\mathrm{i} \delta_{M}(\lambda)\right]
$$

and

$$
r(\lambda)=\sqrt{R(\lambda)} \exp \left[\mathrm{i} \delta_{r}(\lambda)\right]
$$

where $R_{M}(\lambda)$ and $R(\lambda)$ are the wavelength-dependent reflectances of the mirror and the structure, respectively, $\delta_{M}(\lambda)$ and $\delta_{r}(\lambda)$ are the phase changes on reflection from the mirror and the thin-film structure. The phase change $\delta_{r}(\lambda)$ can be represented as the sum of two contributions

$$
\delta_{r}(\lambda)=k(\lambda) 2 n_{1}(\lambda) d+\phi_{n l}(\lambda)
$$

where $k(\lambda)=2 \pi / \lambda$ is the wavenumber, $n_{1}(\lambda)$ is the refractive index of the thin film and $d$ is the thin-film thickness. The first term in Eq. (3) represents the phase change that the reflected wave experiences at normal incidence as it traverses the thin film once from one boundary to the other and back, and $\phi_{n l}(\lambda)$ is a nonlinear phase function due to the multiple reflections within the thin film of the structure $[5,6]$. The nonlinear phase function $\phi_{n l}(\lambda)$ oscillates around the zero value as is demonstrated for a $\mathrm{SiO}_{2}$ thin film on a Si substrate [14]. If the product $n_{1}(\lambda) d$ in Eq. (3) is replaced by the wavelength independent 
term $L_{0}$, we obtain a modified nonlinear phase function $\delta(\lambda)[11,12]$ given by

$$
\delta(\lambda)=\delta_{r}(\lambda)-k(\lambda) 2 L_{0}
$$

which also oscillates around the zero value for a suitably chosen $L_{0}$.

The interference between the beams of the Michelson interferometer is resolved by a spectrometer as the channeled spectrum

$$
I(\lambda)=I_{0}(\lambda)\{1+V(\lambda) \cos [\varphi(\lambda)]\},
$$

where $I_{0}(\lambda)$ is the reference spectrum, $V(\lambda)$ is a visibility term, which depends on the reflectance $R(\lambda)$ of the thin film structure [12], and $\varphi(\lambda)$ is the phase function given by

$$
\varphi(\lambda)=\delta_{M}(\lambda)-\delta_{r}(\lambda)-\delta_{B S}(\lambda)+k(\lambda)\left[2 L_{1}+2 n(\lambda) t_{\mathrm{eff}}\right],
$$

where $\delta_{B S}(\lambda)$ is the phase change owing to the beam splitter, $2 L_{1}$ is the difference of path lengths between the interfering beams in the air whose dispersion is neglected and $n(\lambda)$ is the refractive index of the beam splitter material. The nonlinear phase function $\delta(\lambda)$ is given by

$$
\delta(\lambda)=\delta_{M}(\lambda)-\varphi(\lambda)-\delta_{B S}(\lambda)+k(\lambda)\left[2\left(L_{1}-L_{0}\right)+2 n(\lambda) t_{\mathrm{eff}}\right] .
$$

Because Eq. (7) contains the phase contributions introduced by the beam splitter and the mirror, a reference measurement is required for the extraction of the nonlinear phase function $\delta(\lambda)$ from $\varphi(\lambda)$.

For the reference measurement, the thin-film structure is replaced by a reference sample of the known complex reflection coefficient

$$
r_{r e f}(\lambda)=\sqrt{R_{r e f}(\lambda)} \exp \left[\mathrm{i} \delta_{r e f}(\lambda)\right]
$$

and the interference between the beams of the Michelson interferometer gives rise to the channeled spectrum

$$
I_{\text {ref }}(\lambda)=I_{0 r e f}(\lambda)\left\{1+V_{\text {ref }}(\lambda) \cos \left[\varphi_{r e f}(\lambda)\right]\right\},
$$

where $\varphi_{\text {ref }}(\lambda)$ is the phase function given by

$$
\varphi_{r e f}(\lambda)=\delta_{M}(\lambda)-\delta_{r e f}(\lambda)-\delta_{B S}(\lambda)+k(\lambda)\left[2 L_{2}+2 n(\lambda) t_{\mathrm{eff}}\right]
$$


where $2 L_{2}$ is the difference of path lengths between the interfering beams in the air, which is adjusted as close to $2 L_{1}$ as possible. Using Eqs. (7) and (10), the phase function $\delta(\lambda)$ is given by

$$
\delta(\lambda)=\delta(\lambda ; \Delta L)-k(\lambda) 2 \Delta L
$$

where

$$
\delta(\lambda ; \Delta L)=\varphi_{r e f}(\lambda)-\varphi(\lambda)+\delta_{r e f}(\lambda)-k(\lambda) 2 L_{0}
$$

and $\Delta L=L_{2}-L_{1}$. The channeled spectra (5) and (9) can be processed by a WFT [14] to extract the phase functions $\Phi(\lambda)$ and $\Phi_{\text {ref }}(\lambda)$ with the ambiguity of $m 2 \pi$, where $m$ is an integer. The retrieved (measured) nonlinear phase function of a thin-film structure is given by

$$
\delta^{r}(\lambda ; \Delta L)=\Phi_{r e f}(\lambda)-\Phi(\lambda)+m 2 \pi+\delta_{r e f}(\lambda)-k(\lambda) 2 L_{0} .
$$

If $\Delta L=0$, the nonlinear phase function $\delta(\lambda ; \Delta L)$ is, as indicates Eq. (11), identical with $\delta(\lambda)$. It is desirable to reach $L_{1}$ and $L_{2}$ in the both measurement steps as close as possible to minimize the effect of $\Delta L$ in Eq. (11). A simple procedure to adjust $|\Delta L|<200 \mathrm{~nm}$ can be used (see later).

\section{Numerical example}

To demonstrate the applicability of our new two-step method in retrieving the nonlinear phase function $\delta^{r}(\lambda ; \Delta L)$, we consider light incident on a surface of a thin-film structure represented by a uniform $\mathrm{SiO}_{2}$ thin film of the thickness $d=450 \mathrm{~nm}$ on a $\mathrm{Si}$ substrate. In this case multiple reflections take place and the complex reflection coefficient $r(\lambda)$ of the structure can be expressed according to the well-known relation [1]. To simulate the channeled spectrum $I(\lambda)$ resolvable in the first step, we consider a Michelson interferometer with a cube beam splitter made of pure BK7 optical glass whose effective thickness is $t_{\text {eff }}=-10 \mu \mathrm{m}$. Next, we consider $\delta_{M}(\lambda)=\delta_{B S}(\lambda)=0$. Taking into account the known wavelength dependences for the refractive index $n(\lambda)$ of the pure BK7 optical glass [15], the refractive index of the $\mathrm{SiO}_{2}$ thin film [12] and the complex refractive index of the Si substrate [16], we computed the spectrum $I(\lambda)$ in the range from 350 to $1000 \mathrm{~nm}$ for a suitably chosen $L_{1}$. The corresponding spectral signal $S(\lambda)=I(\lambda) / I_{0}(\lambda)-1$ with added noise is shown in Fig. 2 by the solid line. To simulate the reference channeled spectrum $I_{r e f}(\lambda)$ resolvable in the second step, we consider that the thin-film structure in the Michelson interferometer is replaced by a reference sample represented by a 
bare Si substrate and that path length difference $\Delta L=100 \mathrm{~nm}$. The corresponding spectral signal $S_{r e f}(\lambda)=I_{r e f}(\lambda) / I_{0 r e f}(\lambda)-1$ with added noise is shown in Fig. 2 by the dashed line.

The two spectral signals are processed by a WFT [14] to retrieve the unwrapped phases $\Phi(\lambda)$ and $\Phi_{r e f}(\lambda)$. Then $L_{0}=570 \mathrm{~nm}$ is chosen to obtain the nonlinear phase function $\delta^{r}(\lambda ; \Delta L)$ oscillating around the zero value as is shown in Fig. 3 by the solid curve. This function is compared with the theoretical nonlinear phase function to determine the thickness $d$ of the $\mathrm{SiO}_{2}$ thin film on the Si substrate. The theoretical nonlinear phase $\delta(\lambda)$ is fitted to the retrieved one $\delta^{r}(\lambda ; \Delta L)$ using Eq. (11) and the Levenberg-Marquardt least-squares algorithm [17]. The method determines the maximum-likelihood estimate of parameters $\Delta L$ and $d$ that minimizes the figure-of-merit function $\chi^{2}$, defined by

$$
\chi^{2}(\Delta L, d)=\sum_{i=1}^{N}\left[\delta^{r}\left(\lambda_{i} ; \Delta L\right)-\delta\left(\lambda_{i} ; \Delta L, d\right)\right]^{2}
$$

where $\lambda_{i}$ are wavelengths at which the fit is performed (450 to $900 \mathrm{~nm}$ ). We reached an excellent agreement between the theoretical values and the results of processing with the correlation coefficient as high as 0.99996 and the parameters $\Delta L=100.0 \mathrm{~nm}$ and $d=449.9 \mathrm{~nm}$.

\section{Experimental setup}

The experimental setup used in the application of white-light spectral interferometry to measure the nonlinear phase function of a thin-film structure is shown in Fig. 1. It consists of a white-light source: a halogen lamp HL-2000 (Ocean Optics) with launching optics, a collimating lens, a bulk-optic Michelson interferometer with a cube beam splitter made of BK7 optical glass, a metallic mirror connected to a translation stage with a piezoelectric element (Thorlabs), a thin-film structure, a miniature fiber-optic spectrometer S2000 (Ocean Optics) and other components [13]. The thin-film structure is represented by a uniform $\mathrm{SiO}_{2}$ thin film on a $\mathrm{Si}$ wafer. Four different samples with four different $\mathrm{SiO}_{2}$ thin-film thicknesses were under study as is specified in a previous paper [13]. The $\mathrm{Si}$ wafers were annealed in a furnace at $1200^{\circ} \mathrm{C}$ and four annealing times were selected in order to prepare the $\mathrm{SiO}_{2}$ thin film of four different thicknesses ranging approximately from 300 to $450 \mathrm{~nm}$. The experimental setup is of such parameters that channeled spectra of sufficiently high visibility were recorded for the thin-film structure under study $[11,12]$. 


\section{Experimental results and discussion}

First, the channeled spectrum $I(\lambda)$ is recorded at the output of the Michelson interferometer with the thin-film structure under study when the path length difference $L_{1}$ is adjusted. To obtain the spectral signal $S(\lambda)$, which is needed in retrieving the phase function $\Phi(\lambda)$ by using the WFT, the reference spectrum $I_{0}(\lambda)$, i.e., the spectrum without spectral interference fringes, needs to be recorded. To obtain the reference spectrum, one manually translates the stage with the mirror until spectral fringes disappear completely.

Second, the thin film structure is replaced by a bare Si wafer whose complex reflection coefficient $r_{r e f}(\lambda)$ is known and the channeled spectrum $I_{r e f}(\lambda)$ is recorded at the output of the Michelson interferometer when the path length difference $L_{2}$ is adjusted. To reach $L_{1}$ and $L_{2}$ in the both measurement steps as close as possible, the same mirror displacements from a position with the zero OPD are adjusted. The zero OPD in the interferometer is reached when the interference spectrum coincides with the reference spectrum [18]. We have revealed that this simple procedure enables us to adjust $|\Delta L|<200 \mathrm{~nm}$. The reference spectrum $I_{0 r e f}(\lambda)$ is also recorded in the setup in the same way as in the first measurement step. From the corresponding spectral signal $S_{r e f}(\lambda)$, the phase function $\Phi_{\text {ref }}(\lambda)$ is retrieved. Using the known phase change $\delta_{\text {ref }}(\lambda)$ on reflection from the Si substrate computed from the data available [16], the measured nonlinear phase $\delta^{r}(\lambda ; \Delta L)$ oscillating around the zero value is obtained by using Eq. (13) and a suitably chosen $L_{0}$. The function is compared with the theoretical one in order to determine the thickness $d$ of different samples of the $\mathrm{SiO}_{2}$ thin film on the Si substrate.

Figure 4 shows the comparison of the results of fitting the theoretical nonlinear phase to the measured one for sample 1 using the Levenberg-Marquardt least-squares algorithm presented above. Figure 4 demonstrates very good agreement between theory and experiment with the correlation coefficient as high as 0.99962 and the thin-film thickness $d=285.6 \mathrm{~nm}$. In the same figure is shown the measured nonlinear phase of sample 2 compared with the theoretical one. Once again, very good agreement is confirmed with parameters of the fit: the correlation coefficient as high as 0.99951 and the thin-film thickness $d=338.6 \mathrm{~nm}$.

Next, Fig. 5 shows a comparison of the experimental results with the theoretical ones concerning the nonlinear phase function $\delta^{r}(\lambda ; \Delta L)$ for the remaining two samples. Note that these functions include the artifacts due to the phase retrieval procedure [14]. Figure 5 illustrates very good agreement between theory and experiment and Table 1 lists the corresponding thicknesses $d$ and the correlation coefficients $R$. If we assume that the phase is retrieved with an error of $0.03 \mathrm{rad}$ [14], the thin-film thickness is obtained with an error of 1 
$\mathrm{nm}$. We also measured in the same setup the reflectances of the all samples by using a three-step procedure presented in a previous paper [13] and Table 1 lists the thicknesses $d_{R}$ obtained.

We can conclude from the obtained results that there is very good agreement between experiment and theory. The correlation coefficients $\mathrm{R}$ indicate minimum discrepancy between the real structure and the theoretical model adopted. The correlation coefficients $\mathrm{R}$ show that the best agreement is reached for the first sample and the worst for the third one. Moreover, the results obtained in the same setup by techniques of spectral interferometry and reflectometry agree well.

\section{Conclusions}

We have used a new two-step white-light spectral interferometric method for a precise measurement of a nonlinear phase function of a thin-film structure. The method, which is much simpler compared to the techniques such as $[7,8]$, is based on processing of the recorded channeled spectra to extract phase functions in two steps. One step is with a sample under study and the other step is with a reference sample of known phase change on reflection. The two steps allow one to eliminate the phase errors due to optical elements of the interferometer. Moreover, there is no need to know the effective thickness of a beam splitter cube what substantially simplifies the retrieval of the measured nonlinear phase function compared to a previous method [13].

The feasibility of the method was confirmed in measuring the nonlinear phase function of $\mathrm{a} \mathrm{SiO}_{2}$ thin film on a $\mathrm{Si}$ wafer. The measured phase functions were compared with theoretical ones to determine the thin-film thickness for four samples. We confirmed very good agreement between the theoretical and experimental nonlinear phase functions and between the thicknesses determined in the same setup by techniques of spectral interferometry and reflectometry.

The results obtained serve as an illustration of the feasibility of a simple technique in measuring precisely the nonlinear phase function of a thin-film structure. This method has the primary advantage in its normal incidence configuration over a technique such as ellipsometry. It has potential applications in the phase characterization of complex structures such as multilayer mirrors [19]. This technique and a technique of normal incidence reflectometry, applicable in the same setup, can serve as complements to spectroscopic ellipsometry. 


\section{Acknowledgements}

The research has been partially supported by the Ministry of Education, Youth and Sports of the Czech Republic through grant MSM6198910016 and by the regional grant CZ.1.05/2.1.00/01.0040.

\section{References}

[1] R. A. Azzam, N. M. Bashara, Ellipsometry and Polarized Light, North-Holland, Amsterdam, 1977.

[2] G. E. Jellison, Jr., Thin Solid Films 290-291 (1996) 40.

[3] R. Swanepoel, J. Opt. Soc. Am. A 2 (1985) 1339.

[4] F. Hirth, M. Rössner, M. Jakobi, A. V. Koch, IEEE International Symposium on Optomechatronic Technologies (ISOT 2009), 2009, p. 104.

[5] S.-W. Kim, G.-H. Kim, Appl. Opt. 38 (1999) 5968.

[6] U. Schnell, R. Dändliker, S. Gray, Opt. Lett. 21 (1996) 528.

[7] Y.-S. Ghim, S.-W. Kim, Opt. Express 14 (2006) 11885.

[8] Y.-S. Ghim, S.-W. Kim, Appl. Phys. Lett. 91 (2007) 091903.

[9] H. Xue, W. Shen, P.Gu, Z. Luo, Y. Zhang, X. Liu, Chin. Opt. Lett. 7 (2009) 446.

[10] T. Doi, K. Toyoda, Y. Tanimura, Appl. Opt. 36 (1997) 7157.

[11] P. Hlubina, D. Ciprian, J. Luňáček, M. Lesňák, Opt. Express 14 (2006) 7678.

[12] P. Hlubina, D. Ciprian, J. Luňáček, R. Chlebus, Appl. Phys. B 88 (2007) 397.

[13] P. Hlubina, J. Luňáček, D. Ciprian, R. Chlebus, Appl. Phys. B 92 (2008) 203.

[14] P. Hlubina, J. Luňáček, D. Ciprian, R. Chlebus, Opt. Commun. 281 (2008) 2349 .

[15] Schott Computer Glass Catalog 1.0, Schott Glasswerke, Mainz, 1992.

[16] E. D. Palik, Handbook of Optical Constants of Solids, Academic Press, Orlando, 1995.

[17] Optimization Toolbox for Use with MATLAB, MathWorks, Mass. 2000.

[18] M. M. Brundavanam, N. K. Viswanathan, D. Narayana Rao, Appl. Opt. 47 (2008) 6334.

[19] P. Hlubina, J. Luňáček, D. Ciprian, Opt. Lett. 34 (2009) 1564. 
Table 1

The oxidation time $T$ and the thicknesses $d_{R}$ and $d$ of the $\mathrm{SiO}_{2}$ thin films ( $\mathrm{R}$ is the correlation coefficient)

\begin{tabular}{ccccc} 
Sample & $T(\min )$ & $d_{R}(\mathrm{~nm})$ & $d(\mathrm{~nm})$ & $\mathrm{R}$ \\
\hline 1 & 122 & 285.3 & 285.6 & 0.99962 \\
2 & 212 & 338.0 & 338.6 & 0.99951 \\
3 & 326 & 390.2 & 393.2 & 0.99482 \\
4 & 392 & 450.1 & 449.4 & 0.99943 \\
\hline
\end{tabular}




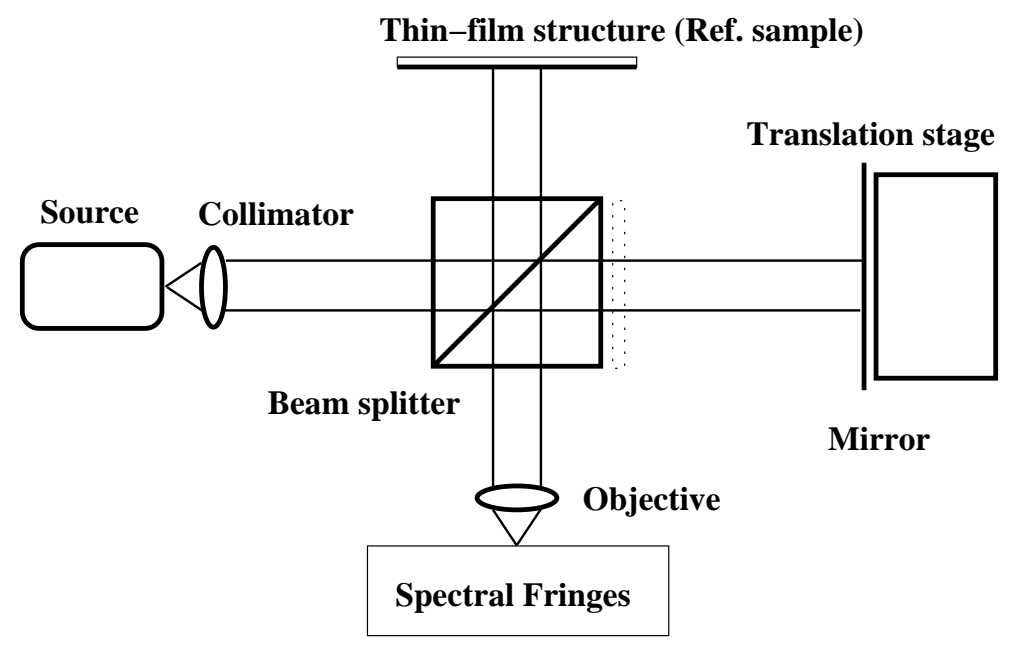

Fig. 1. Experimental setup with a Michelson interferometer to measure a nonlinear phase function of a thin-film structure. 


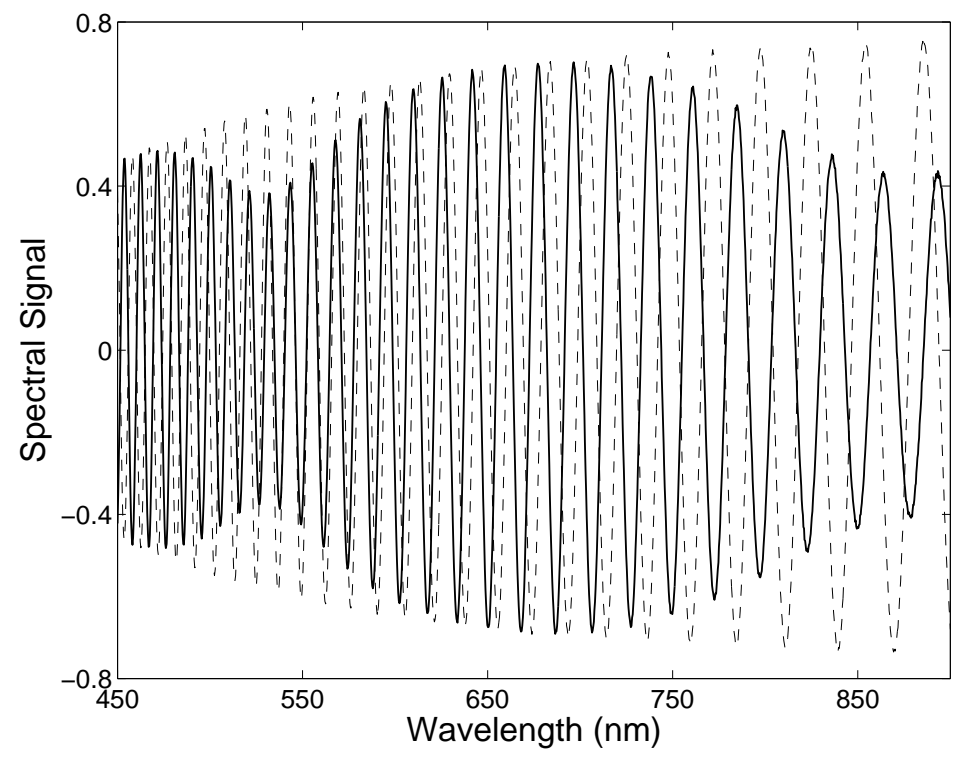

Fig. 2. Theoretical spectral signal with (solid) and without (dashed) the effect of the $\mathrm{SiO}_{2}$ thin film of thickness $d=450 \mathrm{~nm}$ on the Si substrate $(\Delta L=100 \mathrm{~nm})$.

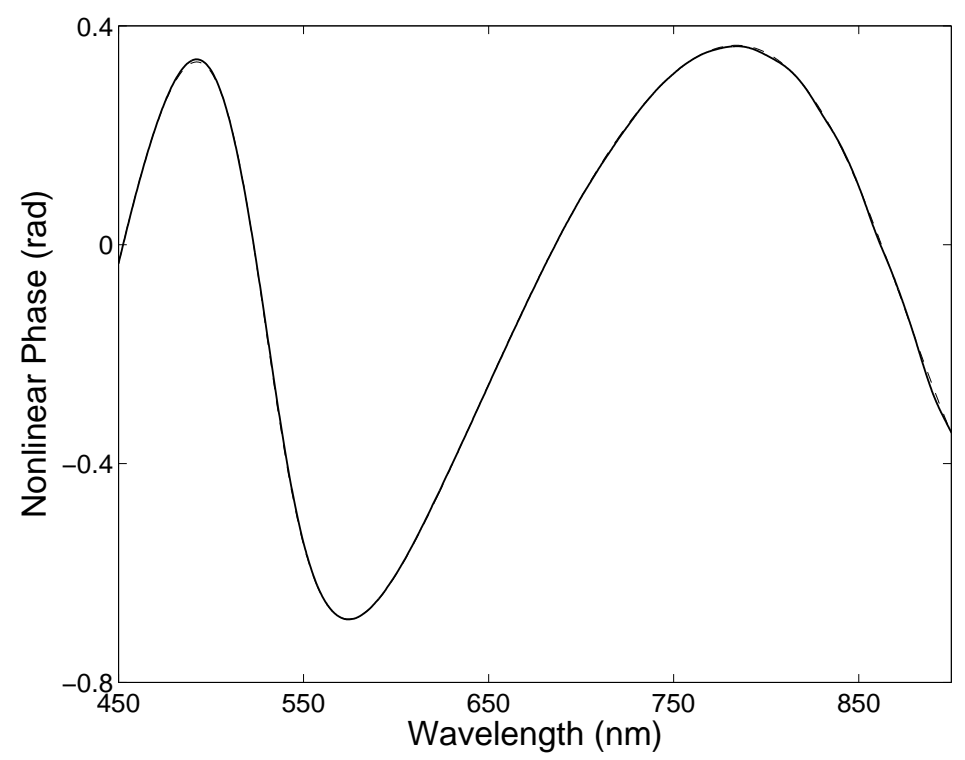

Fig. 3. Nonlinear phase function $\delta^{r}(\lambda ; \Delta L)$ retrieved from the spectral signals shown in Fig. 2. Dashed line is the theoretical function. 


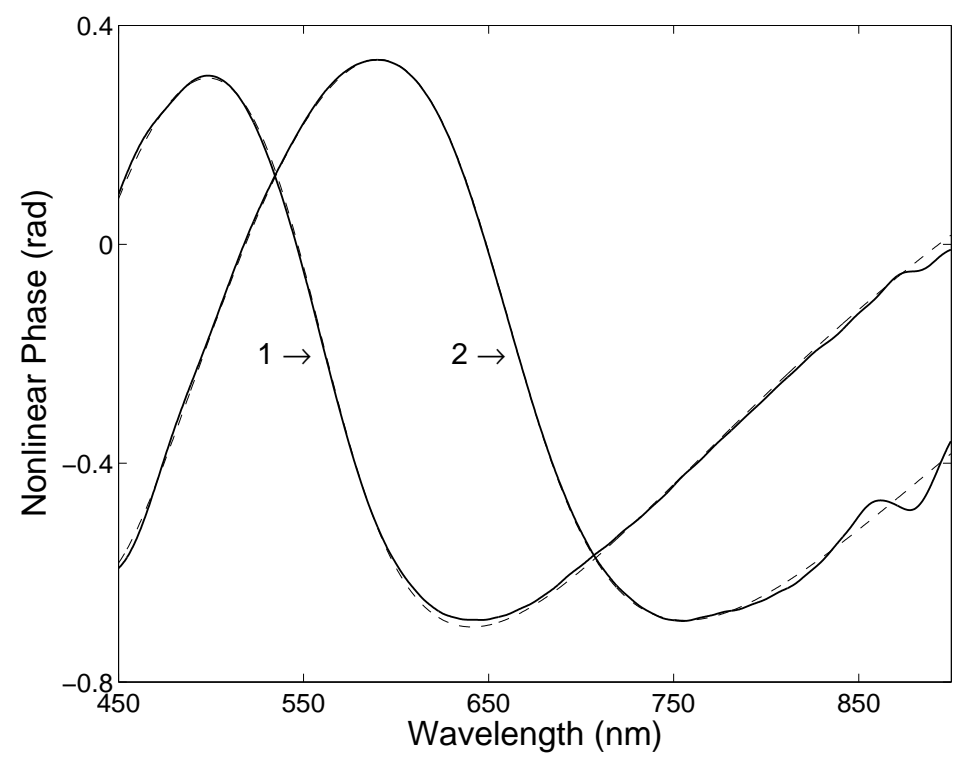

Fig. 4. Measured nonlinear phase as a function of wavelength with the corresponding fit (dashed) for samples 1 and 2.

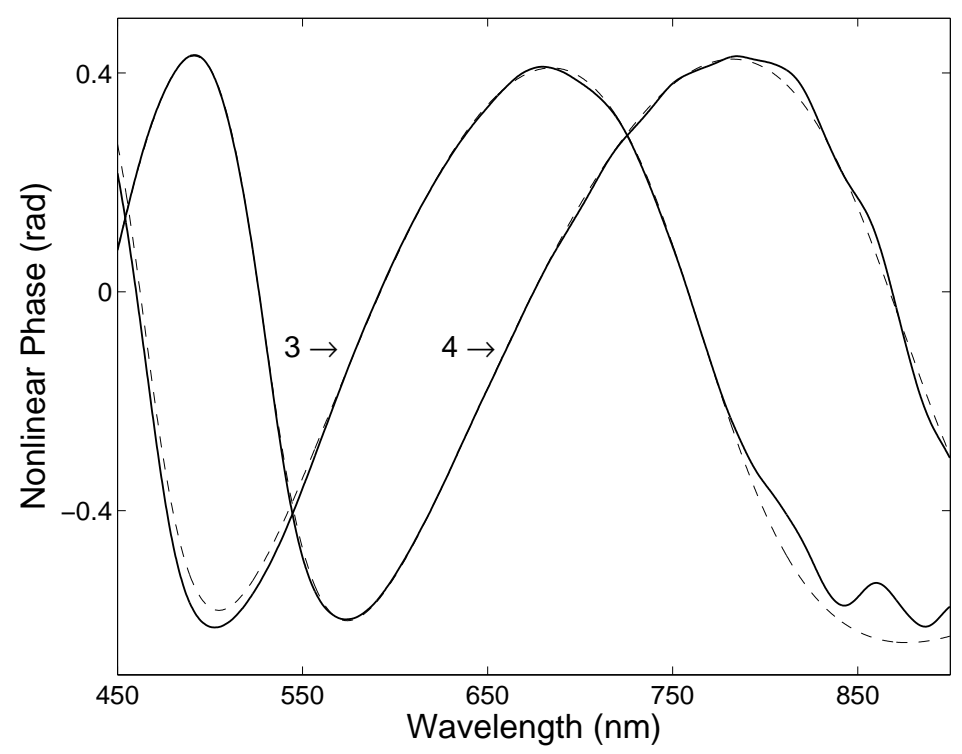

Fig. 5. Measured nonlinear phase as a function of wavelength with the corresponding fit (dashed) for samples 3 and 4. 